\title{
Knowledge, Attitudes and Practices Relating to Fire Prevention among Students in the Elementary Schools of Muang Nakhon Ratchasima, Thailand
}

\author{
Pirutchada Musigapong \\ Institute of Medicine, Suranaree University of Technology \\ Wantanee Phanprasit
}

Faculty of Public Health, Mahidol University

Doi:10.5901/jesr.2013.v3n7p288

\begin{abstract}
In 2010, the department of disaster prevention and mitigation, Thailand reported that during 2006 - 2010 the statistics of fire in temples, schools and offices was increasing every year. Only in 2010, there was 80 cases. The aim of this cross-sectional study was to evaluate knowledge, attitudes and practices (KAP) of the students in thirty two elementary schools toward fire prevention. Verbal consent was taken from the students who were willing to participate in the study. Six hundred and thirty students were 359 female, 266 male and 5 not identified. The mean of age of the studied subjects was $11.09 \pm 1.07$ and ranged from 6 to 13 years. These students completed questionnaire which was designed by the researchers and evaluated by the institute members of occupational health and safety department. The content validity of item-objective congruence index (IOC) is 0.83 which means the consistent questions with the objectives. The collection data were analyzed by 2-independent samples test and Pearson Chi-square $(p<0.05)$. The results indicated that no gender differences were with the level of knowledge, attitudes and practices ( $p=0.072,0.149$ and 0.235 respectively) and the Pearson chi-square showed that the level of knowledge, attitudes was not associated with practices ( $p=0.256$ and 0.572 respectively). The finding also revealed that the students who had not been trained in fire evacuation had more inappropriate behavior or practice and poorer attitude toward fire than those had the experience. Strategy planning to improve attitudes and practices through proper training for fire evacuation among students are needed.
\end{abstract}

Keywords: Knowledge, attitude and practice (KAP), Fire prevention, Primary school

\section{Introduction}

Education quality assurance is the main policy of the government of Thailand to develop and enhance the quality of the education system to be equivalent to international. One of the requirements for the education quality assurance of Thailand define that school need to have an environment and services that encourage learners to develop their full potential including the safety requirement (Ministry of Education, 2011). Although schools have an appropriate and safe environment. In 2010, the department of disaster prevention and mitigation, Thailand reported the statistics of fire in temple, school and official from 1989 to 2010 have totally 2,636 cases which cover all provinces of Thailand. The lowest and highest number of fire is 20 (2004 yr.) and 324 (1992 yr.) cases, respectively (Ministry of Interior, 2010). These data implied that the requirements of education quality assurance concerned with safe environment were inappropriate, improper and not clearly criteria to prevent fire. Therefore, it is necessary to set guidelines for reducing the incident and improving the human potential. Unfortunately, there are no adequate studies about fire prevention of primary school students in Thailand. Therefore, knowing the students' knowledge, attitude and practices fire prevention assist us to improve the guideline of fire prevention in primary school. The purpose of this study was to evaluate knowledge, attitudes and practices of elementary school associated with fire prevention by using method of KAP survey.

A KAP survey is a representative study of a specific population to collect information on what is known, believed and done in relation to a particular topic. A KAP survey gathers information about what respondents know, think and actually do about something or someone. KAP survey can identify knowledge gaps, cultural beliefs, or behavioral patterns that may facilitate understanding and action, as well as pose problems or create barrier (WHO, 2008). In particular, knowledge-attitudes questionnaires have been used to evaluate the effectiveness of information interventions intended to change behavior. (Headrick et al., 1992) (Superko et al., 1988) 


\section{Material and Method}

The study was conducted using a descriptive cross-sectional approach to determine the knowledge, attitudes and practices of students in elementary schools located in Muang, Nakhon Ratchasima during the educational year 2009 2010.

\subsection{Population and Participants}

The researchers surveyed area in Muang, Nakhon Ratchasima to collect the data about the number of primary school and student. The data showed that fifty seven elementary schools were classified in four scales such as small scale (students < 120), medium scale (students $=121-600$ ), large scale (students $=601-1,500$ ) and extra-large scale (students $>1,500$ ) had 22, 29, 2 and 4, respectively. Overall elementary students had 13,608 (population) which were determined sample size by Yamane (1967:886). Yamane provides a simplified formula to calculate sample sizes. A 95\% Confidence level and P-value $=0.5$ are assumed for Equation 1 (Glenn D., 1992).

$$
n=\frac{N}{1+N\left(e^{2}\right)}
$$

Where $\mathrm{n}$ is the sample size, $\mathrm{N}$ is the population, and $\mathrm{e}$ is the level of precision $(\mathrm{e}=0.05)$.

Calculated sample size was 389 subjects, so the number of samples is randomly selected from population and schools. Small and medium-sized schools for the simple random $50 \%$ of all to study a number of 11 and 15 , respectively. Due to two large-sized and four extra-large sized elementary school were included all in this study. So there are thirty two schools that were selected as the study group. Elementary students were invited to participate and were explained the objectives of this study. The researcher obtained verbal consent from all participants.

\subsection{Questionnaire}

The questionnaire was created by the research team, was peer-reviewed and was tested the content validity of itemobjective congruence index (IOC) by three lecturers of occupational safety and health department, Suranaree University of Technology. The average of IOC score was 0.83 . These score indicated that the questions are consistent to the objectives.

The questionnaire was classified into the following four parts: part 1: demographic information such as gender, age, level of education); part 2: knowledge about fire prevention; part 3: attitudes on fire prevention; and part 4: fire prevention practices. The instrument focused on a limited set of questions pertaining to knowledge, attitudes and practices covering important basic aspects of fire prevention. The researchers distributed 640 questionnaires but were given back 630 questionnaires.

\subsection{Knowledge, Attitude and Practice scale}

The knowledge was involved 9 items and attitudes had 8 items about fire, fire-fighting, fire prevention. The practice of fire prevention was investigated using a separate set of 9 questions about fire prevention and 5 questions concerned with risk activity to be fire. The participants completed the questionnaires and selected the correct answers from the MCQ answers. Scoring was done by awarding marks (correct response $=1$, wrong $=0$, don't know $=0$, and not response $=0$ ) for each item of knowledge and attitudes. The practices scale of each item scored a value of 1 for positive practice and 0 for negative or inadequate practice. Knowledge, attitudes and practices scores were assessed as good (>mean+SD), fair (mean-SD to mean+SD) and poor (<mean-SD).

\subsection{Statistical analysis}

The data obtained from the questionnaires were coded, tabulated and analyzed statistically. Percent, means and standard deviation used to describe the scores from the knowledge, attitudes and practices concerned with fire prevention. Two-independent samples test and Pearson Chi-square were used to test for any significant differences and relationship among variables (95\% Confidence Intervals, P-value < 0.05$)$. 


\section{Results}

A total of 630 elementary students participated in this study, 359 (56.98\%) were females, 266 (42.22\%) were males and $5(0.8 \%)$ were not identify. The mean age of participants was $11.41(\mathrm{SD}=5.8)$ and ages ranged between 6 and 13 years. Majority of the students, $230(36.5 \%)$ had sixth elementary school degree, $220(34.9 \%)$ had fifth elementary school degree and 115 (18.3\%) had fourth elementary degree.

Table 1. Demographic Characteristics of elementary school in Muang, NakhonRatchasima, Thailand.

\begin{tabular}{|c|c|c|c|c|c|}
\hline \multicolumn{2}{|c|}{ Characteristics } & $\mathrm{n}$ & $\%$ & Mean \pm SD & Range \\
\hline \multicolumn{4}{|l|}{ Sex } & - & - \\
\hline- & Female & 359 & 56.98 & & \\
\hline - & Male & 266 & 42.22 & & \\
\hline - & Not identify & 5 & 0.8 & & \\
\hline \multicolumn{4}{|l|}{ Age (years) } & $11.09 \pm 1.07$ & $6-13$ \\
\hline- & 6 & 1 & 0.2 & & \\
\hline - & 7 & 5 & 0.8 & & \\
\hline - & 8 & 11 & 1.7 & & \\
\hline - & 9 & 22 & 3.5 & & \\
\hline - & 10 & 115 & 18.3 & & \\
\hline - & 11 & 220 & 34.9 & & \\
\hline - & 12 & 230 & 36.5 & & \\
\hline - & 13 & 22 & 3.5 & & \\
\hline- & Not identify & 4 & 0.6 & & \\
\hline \multicolumn{4}{|l|}{ Education } & - & $1^{\text {st }}-6^{\text {th }}$ degree \\
\hline- & $1^{\text {st }}$ degree & 6 & 1 & & \\
\hline - & $2^{\text {nd }}$ degree & 8 & 1.3 & & \\
\hline - & $3^{\text {rd d degree }}$ & 17 & 2.7 & & \\
\hline - & $4^{\text {th }}$ degree & 96 & 15.2 & & \\
\hline - & $5^{\text {th }}$ degree & 230 & 36.5 & & \\
\hline - & $6^{\text {th }}$ degree & 271 & 4.3 & & \\
\hline- & Not identify & 2 & 0.3 & & \\
\hline
\end{tabular}

In this study, the level of knowledge, attitudes and practices divided into three level such as good/positive, fair and poor/negative. Most elementary students with knowledge, attitudes and practices level were fair as equal 361 (57.8\%), $405(64.8 \%)$ and $356(57 \%)$, respectively. The KAP level of students indicated that the students have average knowledge, attitudes and practices. The KAP level of all, male and female students were shown in Table 2.

Table 2. The average KAP level of all, male and female students.

\begin{tabular}{|cccccccccc|}
\hline \multirow{2}{*}{ KAP Level } & \multicolumn{3}{c}{ All (\%) } & \multicolumn{3}{c|}{ Male (\%) } & \multicolumn{3}{c|}{ Female (\%) } \\
\cline { 2 - 11 } & $\mathrm{K}$ & $\mathrm{A}$ & $\mathrm{P}$ & $\mathrm{K}$ & $\mathrm{A}$ & $\mathrm{P}$ & $\mathrm{K}$ & $\mathrm{A}$ & $\mathrm{P}$ \\
\hline Good/Positive & 26.4 & 12.6 & 22.2 & 30.45 & 10.52 & 24.44 & 23.40 & 14.21 & 20.613 \\
\hline Fair & 57.8 & 64.8 & 57 & 54.89 & 65.04 & 48.87 & 59.89 & 64.62 & 62.952 \\
\hline Poor/Negative & 15.8 & 22.6 & 20.8 & 14.66 & 24.44 & 26.69 & 16.71 & 21.17 & 16.435 \\
\hline
\end{tabular}

Students had the highest score of knowledge question about the extinguishing agents and the lowest score about fire station. The result showed that the students got information from multimedia such as television (391), teacher (326), newspaper (221), internet (162), radio (146), family (146) and placard/flap (79). Therefore, the most media have to attend for providing the knowledge of fire prevention such as television, teacher and newspaper.

Observation regarding KAP questionnaire showed that participants believed that fire is important but don't believe that they were sufficient capability to fire prevention. Furthermore, most students behave incorrectly on fire because there are only a few people (18.8\%) who had practiced fire evacuation. Consequently, data analysis was separate into two groups, the first group of students through a fire evacuation and the other group not trained to indicate the level of knowledge, attitudes and practices that are likely to cause fire (as shown in Table 3 ). 
Table 3. Data of the group of students through a fire evacuation and the other group not trained.

\begin{tabular}{|lll|}
\hline Parameter & 1 $^{\text {st }}$ group* & $2^{\text {nd }}$ group $^{\star *}$ \\
\hline Number of student & $114(18.10 \%)$ & $492(78.10 \%)$ \\
\hline Level of knowledge (avg. score) & Fair (2.09) & Fair $(2.12)$ \\
\hline Level of attitudes (avg. score) & Fair (2.04) & Poor (1.88) \\
\hline Practices & & \\
\hline 1. Smoking & $7(11.1 \%)$ & $21(3.33 \%)$ \\
2. Cooking & $85(13.49 \%)$ & $368(58.41 \%)$ \\
3. Burning trash & $63(10 \%)$ & $232(36.83 \%)$ \\
4. Repairing Electric Appliance & $13(2.06 \%)$ & $40(6.35 \%)$ \\
5. Playing squib/firework & $44(6.98 \%)$ & $220(34.92 \%)$ \\
\hline
\end{tabular}

Number of Missing students $=13$

* group of students through a fire evacuation

** group of students not trained

Information revealed that the second group of students with the level of attitude was worse than the first group. The second group was the practices that can cause a fire, such as smoking, cooking, and repairing electric appliance, than the first group to three times.

Two-Independent samples test was used to test the different of KAP level in two gender (male, female), the results indicated that no gender differences were with the level of knowledge, attitudes and practices $(p=0.072,0.149$ and 0.235 , respectively). Pearson chi-square test showed that the level of knowledge related with attitudes $(p<0.000)$ but the level of knowledge, attitudes was not associated with practices ( $p=0.256$ and 0.572 , respectively).

\section{Conclusion and Recommendation}

In conclusion, most participants (84.2\%) showed an understanding of fire prevention. Based on our finding, gender no differences in knowledge, attitudes and practices level among elementary students were questioned. However, there is a need for improving practices on fire because there were many activities which are likely to cause fire. Therefore, changing participants' behavior in an appropriate direction is more important. The study of Kanouse and Jacoby (1988) indicated that the development of knowledge leads to new attitudes and changes practices which consistent with the approach to solve the problem for this research. Besides changing attitudes and practices, additional training fire evacuation is still necessary for students to take action for reducing the chance of fire.

\section{Acknowledgement}

The authors would like to thank the voluntarily participants of elementary school in Muang, Nakhon Ratchasima and appreciate staff members of Occupational Health and Safety department, Institute of medicine, Suranaree University of Technology to evaluate the KAP questionnaire.

\section{References}

Bettina. M. B., Rochelle R., Lean M., Carolyn J., Theresa A. N. (1999) Knowledge, attitudes, and practices related to fruit and vegetable consumption of high school students. Journal of Adolescent Health 24: 244-250.

Glenn D. (1992) Determining sample size. Retrieve September 25, 2009, from http://www.sut.ac.th/im/data/read6.pdf

Headrick, L. A., Speroff, T., Pelecanos, H. I. and Cebul, R. D. (1992) Efforts to improve complimence with the National Cholesterol Education Program Guidelines. Results of a randomized controlled trial. Archives of Internal Medicine 152, 2490.

Kanouse, D. E. and Jacoby, I. (1998) When does information change practitioners' behavior? International Journal of Technical Assessment in Health Care 4, 27.

Kayode F. O., Akande T. M., Osagbemi G. K. (2005) Knowledge, attitude and practice of breast self examination among female secondary school teachers in llorin, Nigeria. European Journal of Scientific Research, Vol 10; No. 3: 42-47.

Ministry of Education. Bureau of Educating Testing. (2011). Assessment of quality basic education standards for quality assurance within the education institute. Bangkok.

Ministry of Interior. Department of Disaster Prevention and Mitigation. (2010). Statistic Fire situation in Thailand. Retrieve January 5, 2011, from http://61.19.100.58/public/group4/disaster01/data20y/fire20y_5.pdf

Superko, R. H., Desmond, D. A., de Santos, V. V., Vranizan, K. M. and Farquhar, J. W. (1988) Blood cholesterol treatment of community physicians: a major problem. American Heart Journal 116, 849.

World Health Organization. Advocacy, Communication and Social Mobilization for TB control. (2008). A guide to developing knowledge, attitude and practice surveys. Retrieve Sept. 25, 2011, from http://whqlibdoc.who.int/publications/2008/9789241596176_eng.pdf 